\title{
Characterizing surface viscoelastic integrity of ultra-fast photo-polymerized composites: methods development.
}

DOI:

10.1016/j.dental.2020.07.009

\section{Document Version}

Accepted author manuscript

Link to publication record in Manchester Research Explorer

\section{Citation for published version (APA):}

Watts, D., \& Algamaiah, H. (2020). Characterizing surface viscoelastic integrity of ultra-fast photo-polymerized composites: methods development. Dental Materials, 36(10), 1255-1265.

https://doi.org/10.1016/j.dental.2020.07.009

\section{Published in:}

Dental Materials

\section{Citing this paper}

Please note that where the full-text provided on Manchester Research Explorer is the Author Accepted Manuscript or Proof version this may differ from the final Published version. If citing, it is advised that you check and use the publisher's definitive version.

\section{General rights}

Copyright and moral rights for the publications made accessible in the Research Explorer are retained by the authors and/or other copyright owners and it is a condition of accessing publications that users recognise and abide by the legal requirements associated with these rights.

\section{Takedown policy}

If you believe that this document breaches copyright please refer to the University of Manchester's Takedown Procedures [http://man.ac.uk/04Y6Bo] or contact uml.scholarlycommunications@manchester.ac.uk providing relevant details, so we can investigate your claim.

\section{OPEN ACCESS}




\title{
Characterizing surface viscoelastic integrity of ultra-fast photo-polymerized composites: methods development.
}

Short title: Surface integrity of composites.

\author{
David C Watts ${ }^{1,2}$, Hamad Algamaiah ${ }^{1,3}$ \\ ${ }^{1}$ Biomaterials Science, Division of Dentistry, School of Medical Sciences, University of \\ Manchester, UK \\ ${ }^{2}$ Photon Science Institute, University of Manchester, UK \\ ${ }^{3}$ Department of Restorative Dental Sciences, College of Dentistry, King Saud University, Saudi \\ Arabia
}

\section{Corresponding authors:}

Prof. David C Watts

University of Manchester,

School of Medical Sciences and Photon Science Institute

Coupland 3 Building,

Oxford Road,

Manchester M13 9PL, UK

Email: david.watts@manchester.ac.uk

\section{Dr Hamad Algamaiah}

Department of Restorative Dental Sciences,

College of Dentistry,

King Saud University,

Riyadh

Saudi Arabia

Email: dr.algamaiah@gmail.com 


\title{
Characterizing surface viscoelastic integrity of ultra-fast photo-polymerized composites: methods development.
}

\begin{abstract}
Objective: Resin-Composites are now available designed for polymerization using $3 \mathrm{~s}$ of intense light irradiation. The aim was to develop an experimental method to probe their surface viscoelastic integrity immediately following such rapid photo-cure via macroscopic surface indentation under constant stress as a function of time.
\end{abstract}

Methods: Two bulk-fill composites (Ivoclar AG) were studied: Tetric PowerFill (PFill) and PowerFlow (PFlow). Split molds were used to fabricate cylindrical $\{4 \mathrm{~mm}$ (dia) x $4 \mathrm{~mm}\}$ paste specimens, irradiated at $23{ }^{\circ} \mathrm{C}$ at $0 \mathrm{~mm}$ from the top surface with a Bluephase PowerCure LEDLCU, with $3 \mathrm{~s}$ or $5 \mathrm{~s}$ modes, emitting 3 and $2 \mathrm{~W} / \mathrm{cm}^{2}$, respectively. Post-irradiation specimens were immediately transferred to an apparatus equipped with a flat-ended indentor of $1.5 \mathrm{~mm}$ diameter. $14 \mathrm{MPa}$ compressive stress at the indentor tip was applied centrally in $<2$ min and maintained constant for $2 \mathrm{~h}$. Indentation (I) magnitudes were recorded in real-time ( $\mathrm{t}$ ), with I(t) data re-expressed as \% indentation relative to the $4 \mathrm{~mm}$ specimen height. After $2 \mathrm{~h}$, the indentor was unloaded and indentation recovery was monitored for a further $2 \mathrm{~h}$. Parallel sets of measurements were made where indentation was delayed for $24 \mathrm{~h}$. Further measurements were made with more conventional composites: EvoCeram Bulk Fill (ECeram) and Tetric EvoFlow Bulk Fill (EFlow). These were irradiated for $20 \mathrm{~s}$ at $1.2 \mathrm{~W} / \mathrm{cm}^{2}$. Kinetic data were curve-fitted to exponential growth functions and key parameters analyzed by ANOVA and post-hoc tests $(\alpha=0.05)$.

Results: I(t) plots looked initially similar to bulk creep/recovery: rapid deformation plus viscoelastic response; then, upon unloading: rapid (elastic) recovery followed by partial viscoelastic recovery. However, unlike multiply irradiated and stored bulk-creep specimens, the present specimens were exposed to only 3 or $5 \mathrm{~s}$ "occlusal" irradiation; generating "hard" surfaces. Subsequently, during the $2 \mathrm{~h}$ indentation, the polymer matrix network continued to harden and consolidate. Upon initial loading, $\mathrm{I}(\mathrm{t})$ reached $2-3 \%$ indentation, depending upon the formulation. Upon unloading at $2 \mathrm{~h}$, elastic recovery was only ca. $1 \%$. Delayed loading for $24 \mathrm{~h}$, generated $\mathrm{I}(\mathrm{t})$ plots of significantly reduced magnitude. Most importantly, however, the I(t) plots for ECeram and EFlow, after $20 \mathrm{~s}$ irradiation, showed $\mathrm{I}(\mathrm{t})$ magnitudes quite comparable to the PFill and PFlow rapid-cure composites.

\section{Significance:}

Macroscopic indentation creep has been shown to be a workable procedure that can be applied to rapid-cure materials to assess their immediate surface integrity and developing viscoelastic characteristics. The applied stress of $14 \mathrm{MPa}$ was relatively severe and the indentation/recovery profiles of PowerFill materials with only 3 or $5 \mathrm{~s}$ irradiation demonstrated comparability with their established $20 \mathrm{~s}$ cure siblings, evidencing the suitability of the PowerCure system for clinical application.

Keywords: photopolymerization; dimethacrylate; AFCT; RAFT; resin-composite; indentationcreep; stretched-exponential; viscoelasticity; post-cure. 


\section{Introduction}

There are several current trends in the incremental development of dental resin-composites by academic researchers and particularly by the R\&D departments of dental manufacturing companies [1]. Many developments are driven by (a) competition between manufacturers and (b) requested innovations by clinical dentists. One of the items on the 'wish list' of many restorative dentists is more rapid photo curing (photo polymerization) that maintains the reliability of the cure process, assuming that the clinician follows the manufacturers' instructions precisely.

Visible Light Curing (VLC) by means of Light Curing Units (LCUs) has changed in many respects since the 1970s when it was first introduced [2,3]. The major changes were: (i) the early transition from UV to visible blue light curing (wavelengths ca. $470 \mathrm{~nm}$ ) and (ii) the change from filtered multi-wavelength Quartz Tungsten halogen (QTH) LCUs to Light Emitting Diode (LEDLCUs), with either a single LED chip (output at $470 \mathrm{~nm}$ ) or two types of chip (blue: $470 \mathrm{~nm}$ and violet: $410 \mathrm{~nm}$ ) [4]. The latter are effectively wide-spectrum LCUs.

Photo curing requires the active material to incorporate a photo-initiator (PI) with an absorption spectrum that corresponds to the output spectrum of the LCU. Sufficient light irradiance $\left(\mathbf{I}_{\lambda}\right)$ is also necessary (corresponding to the LED emission rate: photons/s). Each photon carries a certain energy given by $\mathbf{h} \boldsymbol{v}$ where $\mathbf{h}=$ Planck's constant and $\mathbf{v}$ is the frequency of the light. The further major variable is the time period of irradiation $(\mathbf{t})$.

The first photo-cured dental resin-composites were considered to require irradiation for $\mathbf{t}=60 \mathrm{~s}$. Subsequent developments have enabled irradiation times to be reduced from $60>40>20>10 \mathrm{~s}$.

Commercial composites exist for which the required time is claimed to be as low as $5 \mathrm{~s}$. And there is now a product - or product system - available for which $\mathbf{t}=3 \mathrm{~s}$ (Ivoclar-Vivadent AG) [5].

These developments have been facilitated by (i) refinements of the photo-initiator system and (ii) boosting the irradiance (or Radiant Emittance; $\mathrm{mW} / \mathrm{cm}^{2}$ ) of the LCUs. The Ivoclar PowerCure system comprises an advanced Bluephase PowerCure LED-LCU able to deliver $3 \mathrm{~W} / \mathrm{cm}^{2}$ for $3 \mathrm{~s}$ irradiation and two specially formulated composites: PowerFill and PowerFlow.

Photo-polymerization of resin-composites rapidly transforms fluid or 'plastic' paste formulations into hard solids within the irradiation period. However, further post-curing is known to occur that increases both surface and bulk mechanical properties over timescales ranging up to a month postirradiation [3, 6]. The 3 s PowerCure irradiation mode should therefore be capable of producing sufficient material properties within 3 seconds that are demonstrably comparable to properties attained by generally equivalent composites after the more conventional $20 \mathrm{~s}$ irradiation.

The experimental challenge is to devise a method to probe mechanical material properties immediately following $3 \mathrm{~s}$ PowerCure irradiation. This requirement rules out time-consuming specimen preparation to fabricate flexural bar specimens, for example. It also rules out bulk compressive creep measurements that typically require $6 \times 4 \mathrm{~mm}$ solid cylindrical specimen that have been well irradiated from multiple directions [7-11]. Nano-indentation might at first sight appear to be a possibility [12-14]. However, this requires polished specimen surfaces and only 
probes a shallow surface layer and a very small indentation area. Hence, with composite materials, anomalies are possible by nano-indentation of particle phases, rather than the organic matrix [15].

In this investigation, we propose a method that is novel in the context of photo-polymerized resincomposites. This involves immediate time-dependent indentation-creep measurements, with a rigid loaded axisymmetric cylindrical flat-ended punch, into a plane photo-polymerized composite surface. Indentation-recovery measurements are also to be made subsequently on the unloaded specimens.

The null hypotheses to be tested are that:

No significant differences exist between indentation /time profiles, during the loading and recovery periods, for $3 \& 5 \mathrm{~s}$ PowerCure composites and conventionally photo-cured comparator composites.

\section{Theoretical Background}

Two distinct levels of theoretical analysis are relevant to this topic: (i) continuum mechanics of indentation into materials exhibiting plastic, elastic and/or viscoelastic behavior; (ii) molecular theory of photo-polymerization.

\section{Continuum mechanics of surface indentation.}

The problem of a rod or pillar loading a "foundation" material is an ancient one, dating back to the temples built in classical Greece, especially when the supporting material was non-rigid soil, with important implications for the safety of foundations. Within the terms of the classical theory of elasticity, the distribution of stress in a solid when deformed by a rigid punch was first considered in 1885 by Boussinesq [16]. Subsequently several alternative solutions were derived, as summarized by Galin [17], particularly by Harding and Sneddon [18] and Sneddon [19, 20].

A central distinction in contact mechanics is between stresses acting perpendicular to the contacting bodies' surfaces (the normal direction) and frictional stresses acting tangentially between the surfaces.

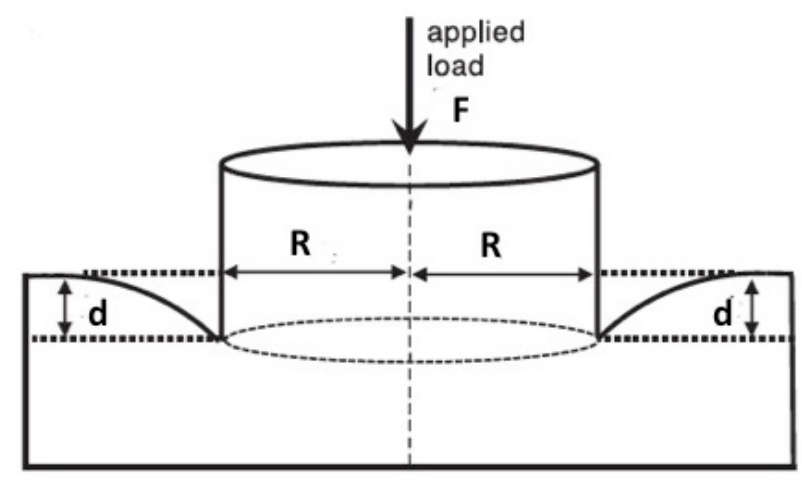

Figure 1: Indentation of a plane surface by a flat-ended cylindrical punch of diameter $\mathbf{2}$ to a depth $\mathbf{d}$ under an applied axial load, F. 
For a cylindrical flat-ended punch indentation the contact geometry is relatively simple because there is no pile-up at the contact edge. The radius of the contact remains fixed and equal to the radius, $\mathbf{R}$, of the punch (Figure 1). When it is pressed into the free surface of an elastic solid, the relationship between the indentation depth $(\mathbf{d})$ and the normal compressive force $(\mathbf{F})$ is given by the linear relationship, [21] (p.117):

$$
\mathrm{F}=2 \mathrm{R} \cdot \mathrm{E}^{*} \cdot \mathrm{d} \quad \text { Equation } 1
$$

where the reduced (or combined) modulus, $\mathrm{E}^{*}$ is given by

$$
\frac{1}{E^{*}}=\frac{1-v_{1}^{2}}{E_{1}}+\frac{1-v_{2}^{2}}{E_{2}} \quad \text { Equation 2 }
$$

$E_{1}$ and $E_{2}$ are the elastic moduli of the test material and rod, and $v_{1}$ and $v_{2}$ are their Poisson's ratios.

If these 4 parameters remain constant, together with force $\mathbf{F}$, then indentation depth (d) will also be constant and time invariant. However, if the modulus $\left(\mathbf{E}_{\mathbf{1}}\right)$ of an elastic test material were to increase (e.g) due to progressive curing, then indentations into successive specimens would exhibit a progressive reduction in elastic indentation depth, under constant load.

The present investigation is concerned with a specific strain history, wherein the creep (progressive deformation) is measured in response to an applied step function of stress, (or loading force). That is, the materials are subjected to an approximately instantaneous loading - and subsequent unloading. The applied stress $\sigma$ at time $t=0$ is:

$$
\sigma_{0}=F / A=F / \pi \cdot R^{2} \quad \text { Equation } 3
$$

The strain $\varepsilon(t)$ in a viscoelastic material will increase with time. The creep compliance, $\mathbf{J}(\mathrm{t})$, is defined as the ratio:

$$
J(t)=\mathcal{E}(t) / \sigma_{0} \quad \text { Equation } 4
$$

The relaxation magnitude, in the compliance formulation, from "start" (0) to "finish" $(\infty)$ over the experimental time range is $\left(\mathrm{J}_{\infty}-\mathrm{J}_{0}\right)$.

In linear viscoelastic materials, the creep compliance is independent of stress level. Under those circumstances, constitutive equations may be developed using the Boltzmann superposition principle, which is a statement of linearity: that the effect of a compound cause is the sum of the effects of individual causes. However, particularly in the case of indentation creep, the assumption of linearity may not hold making the scope for exact analysis severely circumscribed, [21, 22].

Nevertheless, a range of models have been devised to account for viscoelastic behavior. These commonly incorporate various serial or parallel combinations of elastic and viscous 'elements', rather like the spring and damper systems of a motorcar. The springs are assumed to be perfectly 
elastic. The dashpot element may be envisaged as a piston-cylinder assembly in which motion of the piston causes a viscous fluid to move through an aperture. However, real materials are not generally describable by models containing a small number of springs and dashpots.

The simplest pair of models, due to Voigt and Maxwell respectively, consist of a spring and dashpot - arranged either in parallel or in series. Somewhat more realistic behavior can be modeled by the standard linear solid, which contains three elements (Figure 2):

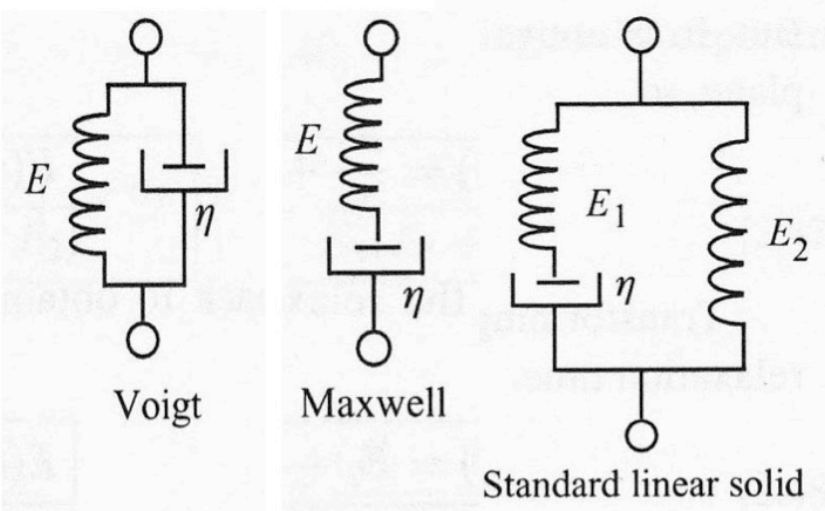

Figure 2: Mechanical model systems consisting of series and/or parallel combinations of elastic and viscous elements.

For the Voigt (or Kelvin) model (Figure 2), both the spring and the dashpot experience the same deformation, or strain, and the total stress is the sum of the stresses in each element. From this, it can be shown [22] (p. 55f) that the creep response is:

$$
J(t)=\frac{1}{E}\left(1-e^{-t / \tau_{c}}\right) \quad \text { Equation } 5
$$

In which $\tau_{c}=\eta / E$ is referred to as the retardation time.

A single exponential relaxation or retardation function undergoes most of its change over about one decade (a factor of 10) in time scale. However, real materials creep or relax over many decades of time.

There is a more general and rather ubiquitous expression for a normalized relaxation processes: the stretched exponential or KWW (after Kohlrausch, Williams, Watts) form [23, 24] with a fractional exponent: $0<\beta \leq 1$.

$$
\phi(t)=e^{-(t / \tau)^{\beta}}
$$

\section{Equation 6}

$\tau$ is the relaxation (or characteristic) time.

A corresponding normalized stretched-exponential growth process is represented by:

$$
1-\phi(t)=\left\{1-e^{-(t / \tau)^{\beta}}\right\}
$$


Dynamic or frequency domain behavior, corresponding to this relaxation function, can be obtained analytically for $\beta=0.5$ [24]. For general values of $\beta$ numerical methods are available [25].

Complex materials with strongly interacting constituents often exhibit KWW behavior [26 - 29]. Crosslinked dental resin-composites incorporate strongly interacting constituents and so this functional form is likely to be applicable to the indentation creep behavior.

Indentation data can be presented either as absolute depth values ( $\mathrm{mm}$ or $\mu \mathrm{m})$ or as percentages of the specimen thickness. In the present work, we will use the latter option for the time-dependent growth of $\boldsymbol{I}(\mathrm{t})$.

$$
I(t)_{\%}=A \cdot\left\{1-e^{-(t / \tau)^{\beta}}\right\} \quad \text { Equation } 8
$$

or

$$
I(t)_{\%}=A .\left\{1-e^{-(t / \tau)^{\beta}}\right\}=A .\left\{1-e^{-\left(t^{\beta / T)}\right.}\right\} \quad \text { Equation } 9
$$

where $A$ corresponds to what would be the magnitude of $I(t) \%$ as $t \rightarrow \infty$.

Again, the parameter $\tau$ is the characteristic time. $T=\tau^{\beta}$ is an alternative representation. The fractional exponent $\beta$ mathematically stretches the viscoelastic process over time (Figure 3 ), away from a simple exponential change, known as Debye behavior, for which $\beta=1$.

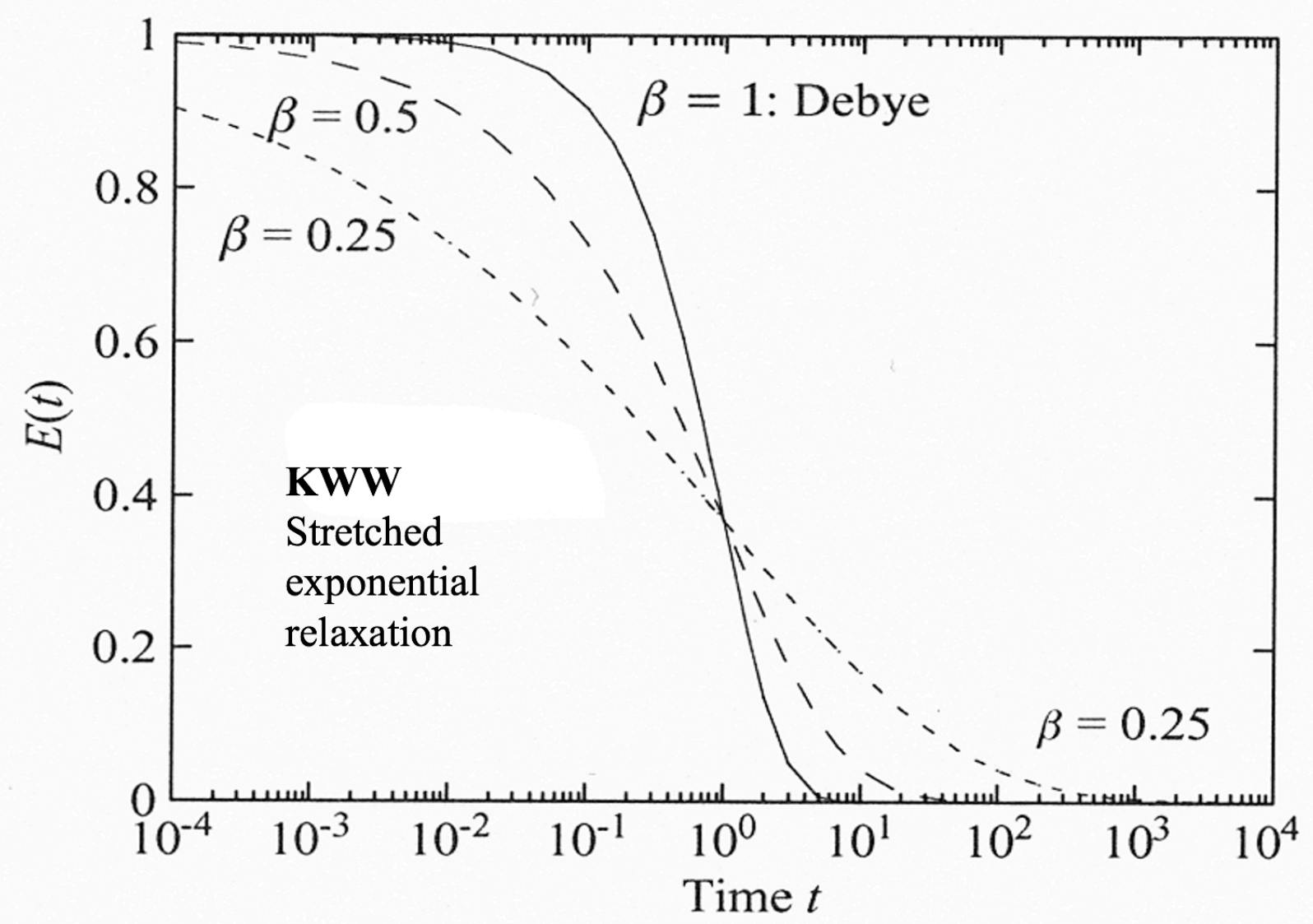

Figure 3: Stretched exponential relaxation functions (cf. Equation 6). Arbitrary units of (eg) modulus and $\left(\log _{10}\right)$ time. As $\beta$ reduces, the relaxation process is increasingly stretched over the time scale. 
For this KWW approach, an expression for the rate of indentation $(\% / \mathrm{s})$ can be obtained by differentiation of equation 7 :

$$
\frac{d I}{d t}=(\beta / \tau) \cdot(t / \tau)^{\beta-1} \cdot e^{-(t / \tau)^{\beta}}
$$

Equation 10

\section{Molecular theory of photopolymerization}

The molecular theory of free-radical photopolymerization has been widely discussed in both the polymer and dental literature, focusing especially upon the characteristics of multi-methacrylate monomers and the resulting cross-linked networks that form the matrix of resin-composites $[6$, 30]. Particular attention has been given to photoinitiator systems and the effects of varying lightirradiation protocols [5, 31-33]. A more recent development has been the possibility of incorporating chain-transfer agents, particularly of the reversible addition fragmentation (AF) type, variously denoted by the acronyms RAFT or AFCT [34]. One such agent is $\beta$-allyl sulfone (Figure 4). This becomes incorporated in the growing network and can modulate the inherently uncontrolled radical chain-reaction so that it proceeds more like a step-growth polymerization and results in a more homogenous network structure [35].<smiles>C=C(CS(=O)(=O)c1ccc(C)cc1)C(=O)OCC</smiles>

Figure 4. $\beta$-allyl sulfone AF chain transfer reagent. 


\section{Materials and Methods}

The experimental design concerned four materials (Table 1). The independent variables were material, curing mode and post-irradiation time. The measurement times began either "immediately" or $24 \mathrm{~h}$ post-irradiation. A total of 48 specimens ( $=4$ /group) were required.

Table 1: Materials Investigated: manufactured by Ivoclar-Vivadent AG, Liechtenstein

\begin{tabular}{|c|c|c|c|c|}
\hline $\begin{array}{l}\text { Material } \\
\text { Codes }\end{array}$ & Product Name & $\begin{array}{c}\text { Lot } \\
\text { Number }\end{array}$ & Resin Matrix* & $\begin{array}{l}\text { Filler Load } \S \\
\%(w t) ;(\text { vol })\end{array}$ \\
\hline PFill & Tetric PowerFill & W92823 & \multirow{2}{*}{$\begin{array}{l}\text { Bis-GMA, Bis-EMA, } \\
\text { UDMA, Aromatic } \\
\text { Dimethacrylate, DCP }\end{array}$} & $79 ; \mathrm{NA}$ \\
\hline PFlow & Tetric PowerFlow & WM1175 & & 71 ;A \\
\hline ECeram & Tetric EvoCeram Bulk Fill & U53769 & $\begin{array}{l}\text { Bis-GMA, Bis-EMA } \\
\text { and UDMA }\end{array}$ & $80 ; 61$ \\
\hline EFlow & Tetric EvoFlow Bulk Fill & U42390 & $\begin{array}{l}\text { Bis-GMA, Bis-EMA, } \\
\text { TCDD }\end{array}$ & $68.2 ; 46.4$ \\
\hline \multicolumn{5}{|c|}{$\begin{array}{l}\text { *Matrix Monomers: Bis-GMA: bisphenol-A-diglycidyl dimethacrylate; } \\
\text { Bis-EMA: bisphenol-A-polyethylene-glycol-diether dimethacrylate; } \\
\text { UDMA: urethane dimethacrylate, }\end{array}$} \\
\hline \multicolumn{5}{|c|}{$\S$ Filler percentages as reported by the manufacturer. } \\
\hline
\end{tabular}

Specimen Preparation:

Stainless steel (SS) split molds were used to fabricate cylindrical-shaped composite paste specimens - each $4 \mathrm{~mm}$ diameter and $4 \mathrm{~mm}$ thick. For each specimen, the resin composite material was placed in the mold, covered with Mylar strip then pressed by a glass slab to ensure a smooth surface and to eliminate any voids. The glass slab was then removed to facilitate close proximity of the light-curing tip above the composite paste surface (covered by Mylar strip).

The specimen was then irradiated directly from the top "occlusal" surface in accordance with a selected Irradiation Protocol (Table 2). The power density of each LCU was verified for each set of specimens using a radiometer (MARC ${ }^{\mathrm{TM}}$ Light Collector, Blue-light Analytics Inc., Halifax, NS, Canada).

Table 2: Irradiation Protocols with two Light-Curing Units (LCUs):

P-Cure = Bluephase PowerCure (Ivoclar Vivadent AG); S10=Elipar S10 (3M).

\begin{tabular}{|c|c|c|c|}
\hline LCU Code & Irradiance $\left(\mathrm{W} / \mathrm{cm}^{2}\right)$ & Irradiation Time (s) & Material Codes \\
\hline P-Cure & 3 & 3 & PFill, PFlow \\
\hline P-Cure & 2 & 5 & PFill, PFlow \\
\hline S10 & 1.2 & 20 & ECeram, EFlow \\
\hline
\end{tabular}


Following irradiation, each specimen was transferred within its SS mold for indentation loading either "immediately" (within $2 \mathrm{~min}$ ) or at $24 \mathrm{~h}$ post-irradiation. The " $24 \mathrm{~h}$ " specimens were stored dry at $37^{\circ} \mathrm{C}$ in the dark.

\section{Indentation Measurement}

The indentation equipment was designed to measure the viscoelastic properties and recovery of resin composites under a static indentation stress. This was achieved by modification of an apparatus for measurement of bulk compressive creep and recovery, as described previously [8, 9 , $11]$.

All specimens were measured within their SS molds at $23 \pm 1{ }^{\circ} \mathrm{C}$. A mold containing a specimen was placed on a raised metal platform. A vertical loading rod, terminating in a $1.5 \mathrm{~mm}$ diameter cylindrical indentor punch (Figure 5 a), was axially aligned and centralized over the specimen surface (Figure $5 \mathrm{~b}$ ). This rod also carried a platform that contacted a calibrated LVDT transducer to detect and record strain changes in the specimens (Figure $5 \mathrm{c}$ ). A cantilever arm could be rapidly raised or lowered onto the top of the loading rod to transfer load to generate compressive stress. The cantilever load was selected to generate, via the indentor punch, a stress of $14 \mathrm{MPa}$.
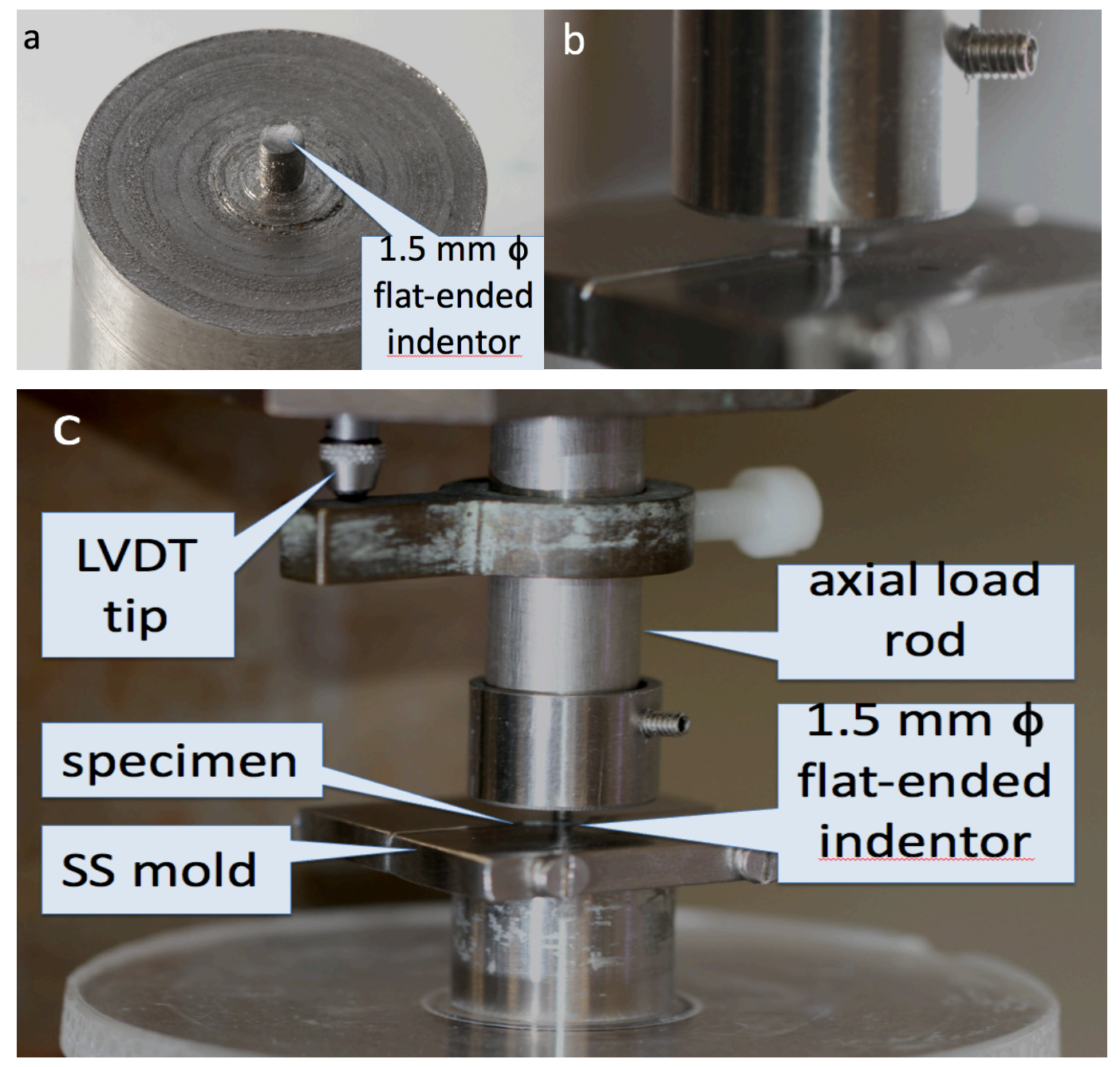

Figure 5: Configuration of the indentation-creep instrument: (a) the indentor punch; (b) the indentor in contact with the surface of a specimen contained within its stainless-steel split mold; (c) the assembly mounted on a fixed platform with the transducer tip registering axial movement of the load rod and indentor punch. 
Once the specimen was placed in position a static force was applied, via the axial rod and the cantilever loading system (not shown), for a period of $2 \mathrm{~h}$. After $2 \mathrm{~h}$, the load was removed and changes in the indentation depth were monitored for a further $2 \mathrm{~h}$.

The LVDT $\mathrm{mV}$ signals were amplified and sent to an analogue-to-digital converter and computer/software (PicoLog 6, Pico Technology, Hardwick, Cambridge, UK). These signals were converted firstly into $\mu \mathrm{m}$ displacement $\left(\Delta_{\mathrm{t}}\right)$, via a calibration coefficient, and then secondly into percentage indentation depth (relative to specimen height: $4,000 \mu \mathrm{m}$ ).

$$
\mathrm{I}(\mathrm{t}) \%=\left[\Delta_{\mathrm{t}} / \mathrm{L}_{\mathrm{o}}\right] \times 100 \quad \text { Equation } 11
$$

$\mathrm{L}_{\mathrm{o}}$ is the specimen height and $\Delta_{\mathrm{t}}$ is the indentation $(\mu \mathrm{m})$.

\section{Statistical and Graphical Analysis}

Sample size calculations were made with $\mathrm{G}^{*}$ power software (V. 3.1.3; Heinrich Heine University, Germany) based on a pilot study. A sample size: $\mathrm{n}=4$ was sufficient to give a power of $80 \%$.

Each of the $(n=4)$ datasets per material, irradiation and loading condition were averaged and plotted. Dynamic curve fitting to the indentation/time plots was achieved using SigmaPlot software (ver. 14).

SPSS 22.0 software (IBM, New York) was used for statistical analysis of key parameters. Normality of distribution was confirmed by the Shapiro-Wilk test. Two-way ANOVA was used for maximum and residual indentation (\%) at both post-irradiation times showing a significant interaction $(\mathrm{p}<0.05)$. Therefore, one-way ANOVA and Tukey post-hoc tests were applied. Irradiation protocols were compared via independent sample t-tests $(\alpha=0.05)$. 


\section{Results}

The indentation versus time plots during the $2 \mathrm{~h}$ loading period were curve fitted, with the KWW stretched exponential function (equation 8) generating close fits for immediate indentation. By contrast, a simpler exponential growth function (equation 12) was more appropriate for modeling the $24 \mathrm{~h}$ delayed loading data. The associated parameters are shown in Tables 3 and 4 and the specific plots and equations for the critical case of PFill, after $3 \mathrm{~s}$ irradiation, are presented in Figure 6.

$$
I(t)_{\%}=y_{0}+a \cdot\left\{1-e^{-b \cdot t}\right\}
$$

\section{Equation 12}

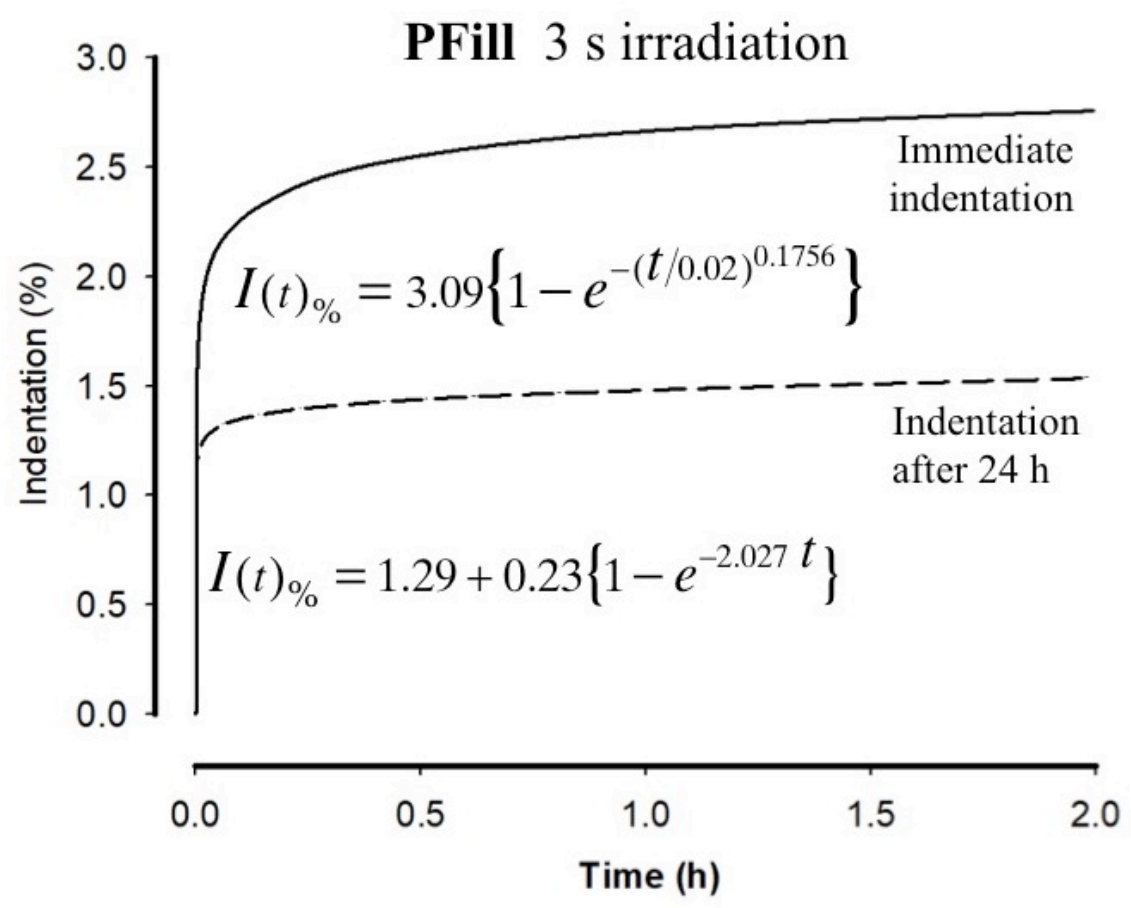

Figure 6: KWW stretched exponential analysis of immediate indentation creep response of PFill, irradiated for $3 \mathrm{~s}$, (upper curve) and simpler exponential analysis of response after $24 \mathrm{~h}$ delayed indentation (lower curve).

Table 3: KWW stretched-exponential parameters for immediate indentation-creep response.

\begin{tabular}{|l|c|c|c|c|}
\hline \multicolumn{1}{|c|}{ Material } & A & $\begin{array}{c}\text { Time constant: } \\
\boldsymbol{\tau}\end{array}$ & $\begin{array}{c}\text { Stretching } \\
\text { exponent: } \boldsymbol{\beta}\end{array}$ & $\mathbf{R}$ \\
\hline PFill 3s & 3.09 & 0.0201 & 0.1756 & 0.9981 \\
\hline PFill 5s & 2.91 & 0.0122 & 0.2187 & 0.9976 \\
\hline PFlow 3s & 3.92 & 0.0103 & 0.2303 & 0.9969 \\
\hline PFlow 5s & 2.56 & 0.0132 & 0.2235 & 0.9987 \\
\hline ECeram 20s & 2.70 & 0.0080 & 0.1731 & 0.9972 \\
\hline EFlow 20s & 3.52 & 0.0081 & 0.1596 & 0.9953 \\
\hline
\end{tabular}


Table 4: Exponential growth parameters for $24 \mathrm{~h}$ delayed indentation-creep response.

\begin{tabular}{|l|c|c|c|c|}
\hline \multicolumn{1}{|c|}{ Material } & yo & a & b & R \\
\hline PFill 3s & 1.29 & 0.23 & 2.0272 & 0.9332 \\
\hline PFill 5s & 1.50 & 0.26 & 2.0772 & 0.9336 \\
\hline PFlow 3s & 1.53 & 0.29 & 2.1434 & 0.9339 \\
\hline PFlow 5s & 2.39 & 0.95 & 4.3164 & 0.9451 \\
\hline ECeram 20s & 1.21 & 0.18 & 2.4531 & 0.9123 \\
\hline EFlow 20s & 1.35 & 0.28 & 2.1579 & 0.9413 \\
\hline
\end{tabular}

Indentation (\%) versus time (h) for each material, irradiation time and measurement time are plotted in Figures 7 (a)-(f).

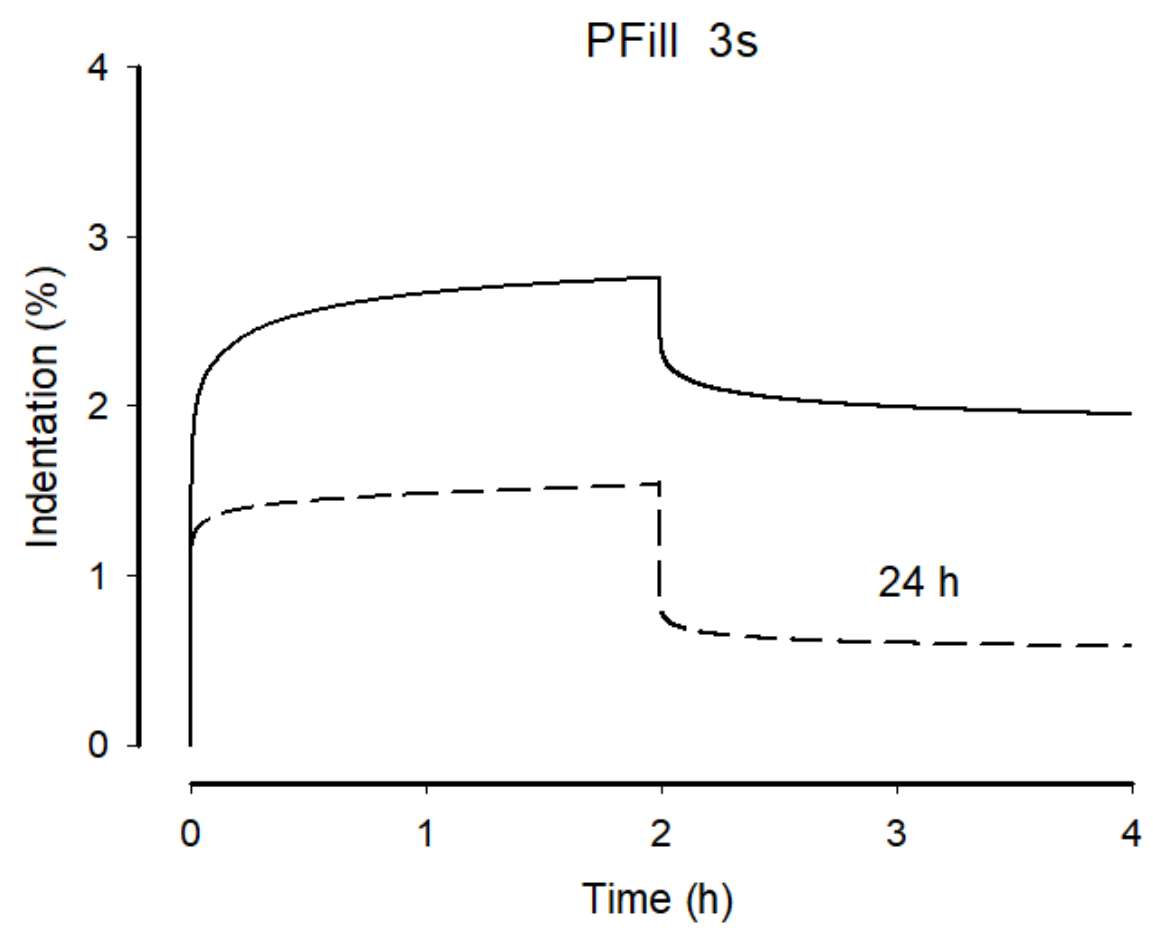

Figure 7 (a) 


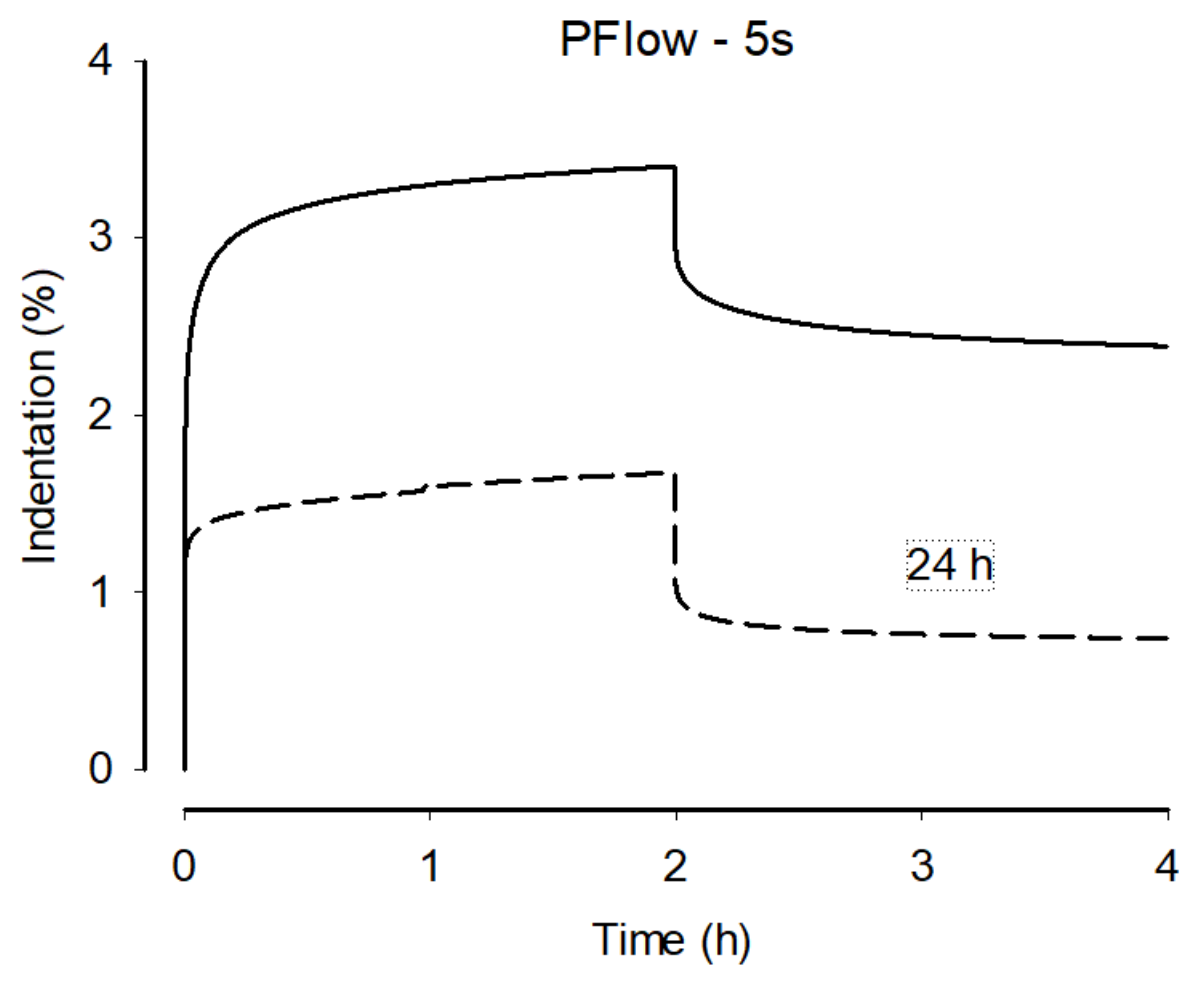

Figure 7 (b)

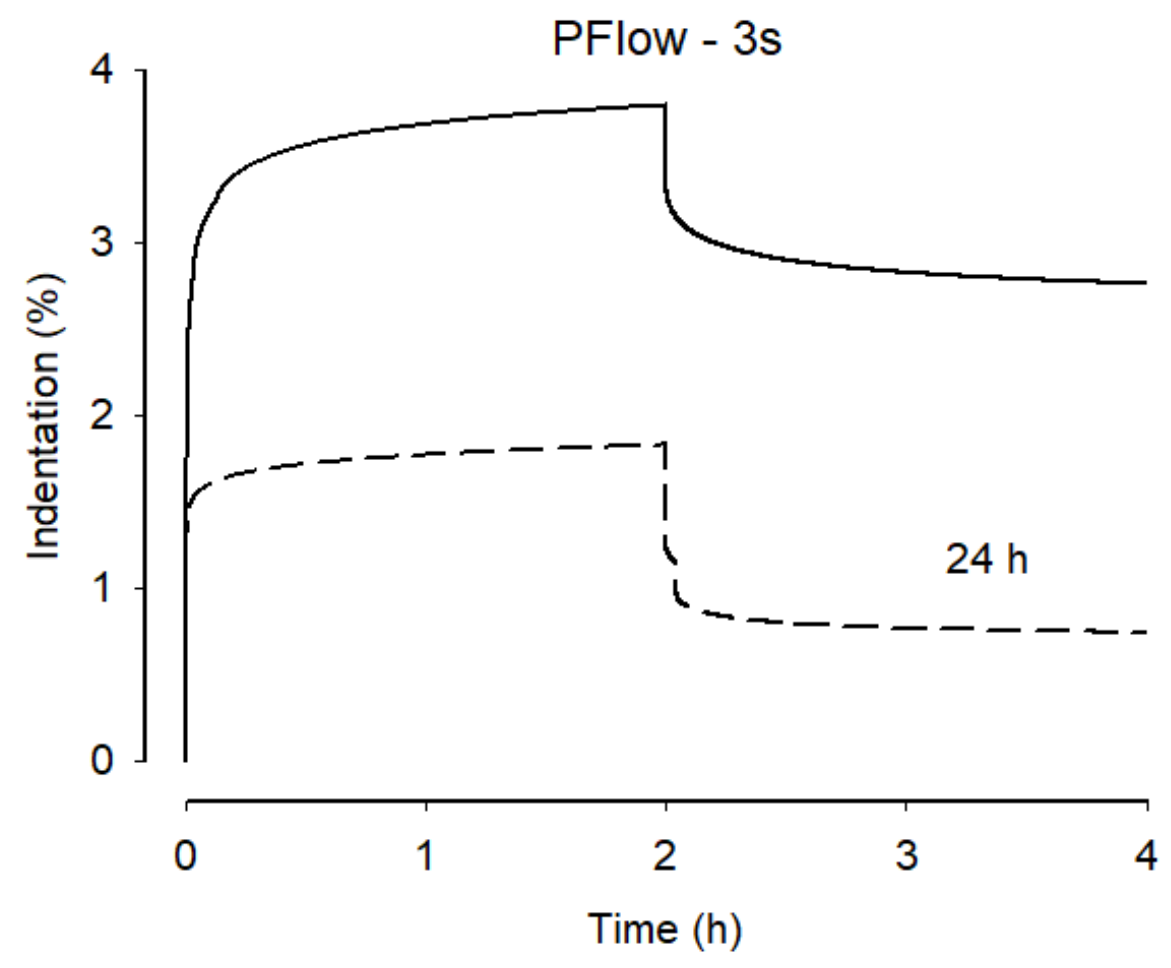

Figure 7 (c) 


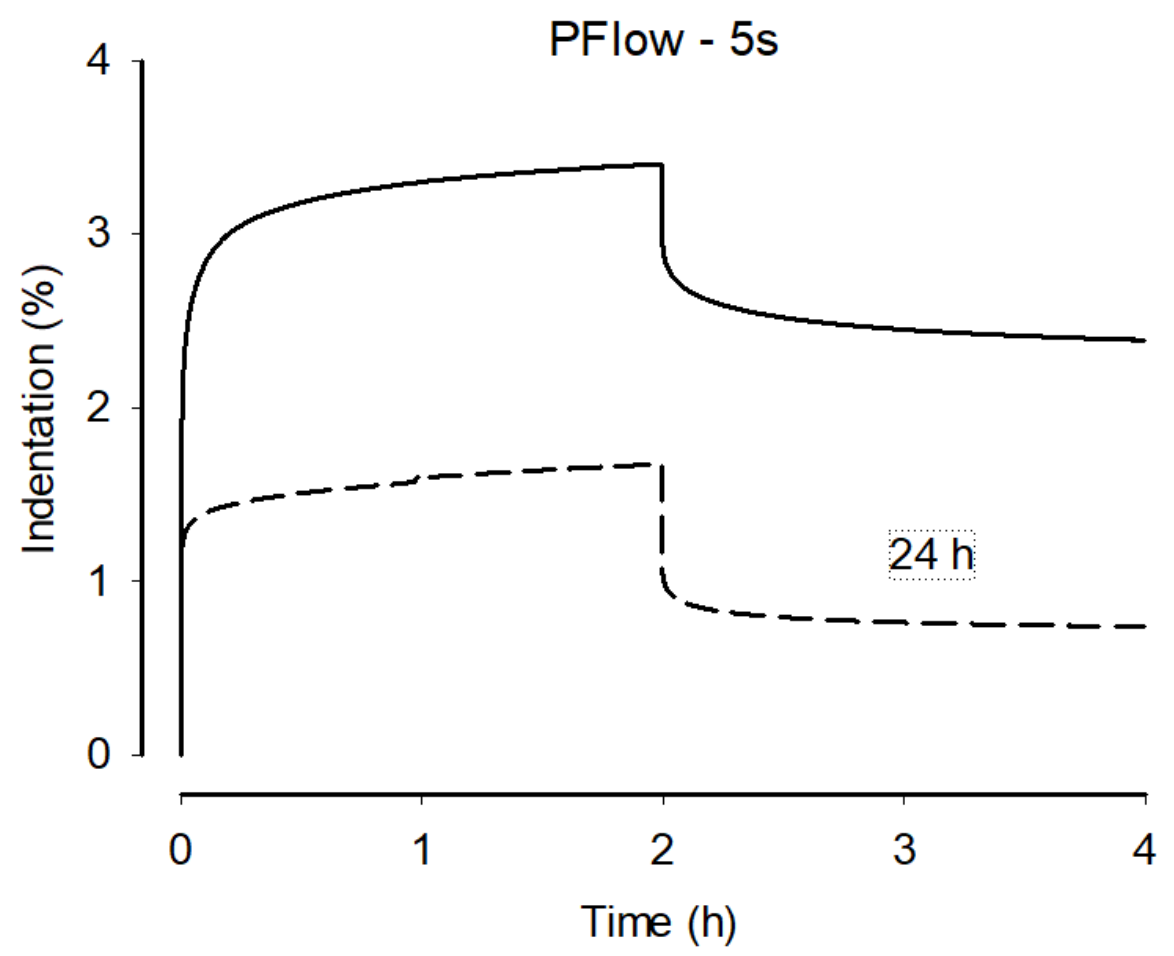

Figure 7 (d)

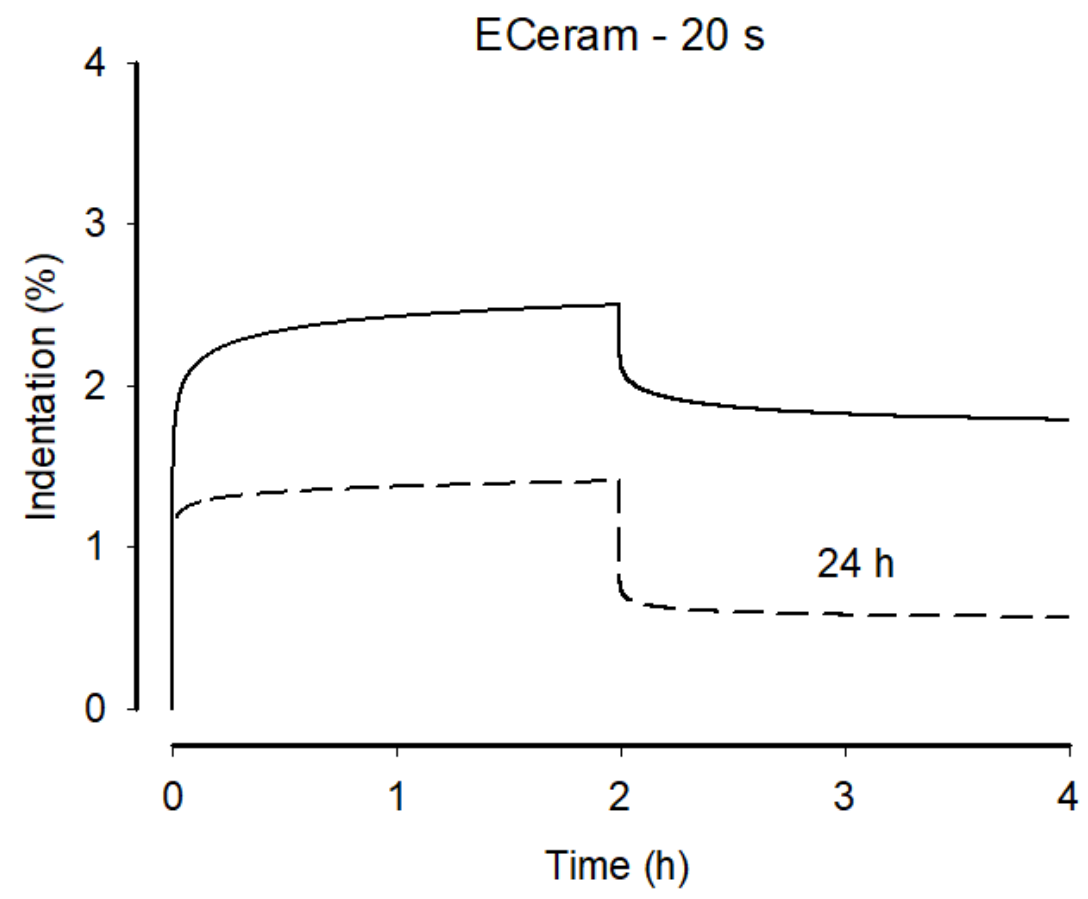

Figure 7 (e) 


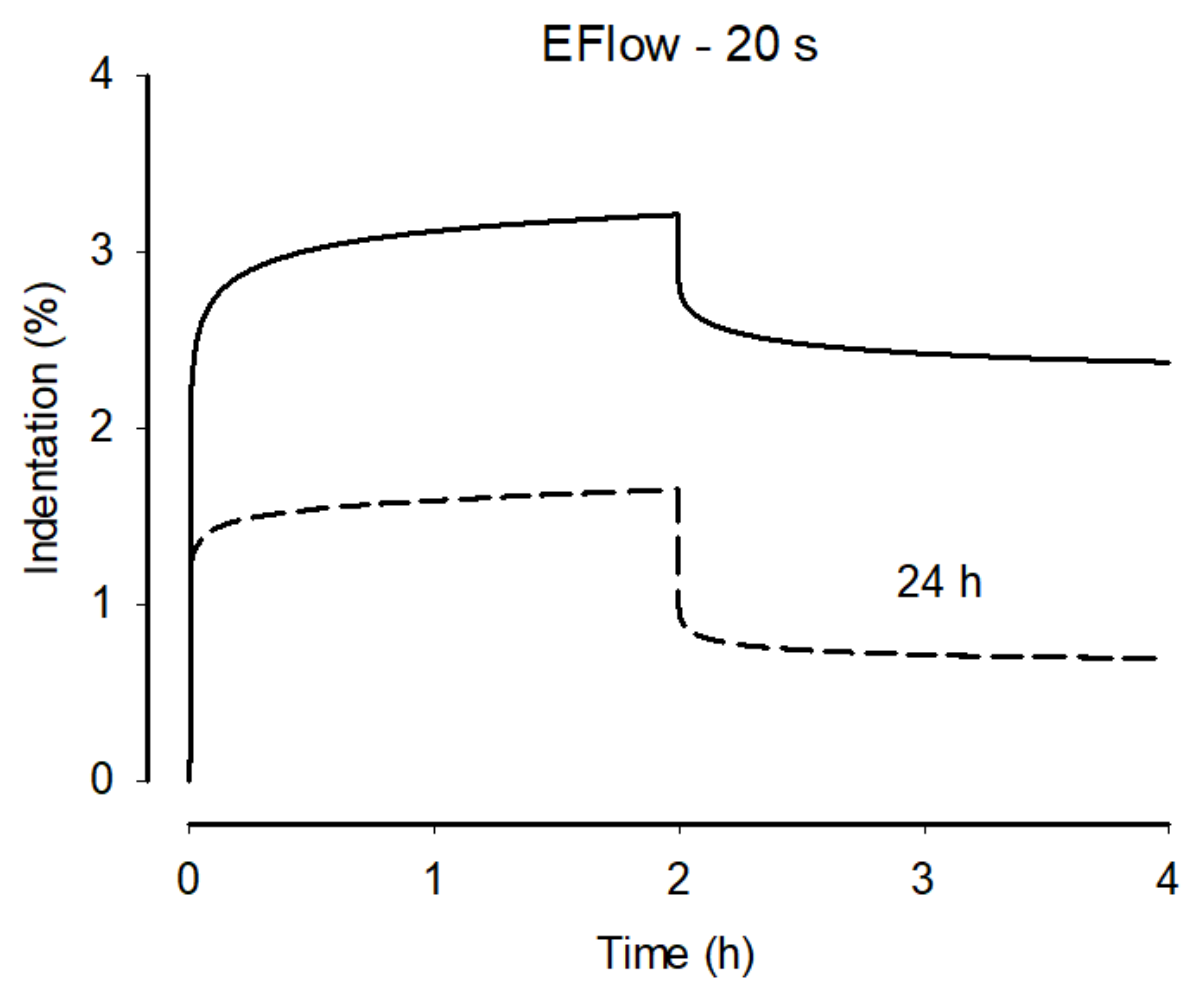

Figure 7 (a) - (f): Indentation as a function of time under $14 \mathrm{MPa}$ stress for $2 \mathrm{~h}$ and unloaded recovery for $2 \mathrm{~h}$ for materials loaded both immediately (within $2 \mathrm{~min}$ - solid curves) and $24 \mathrm{~h}$ after irradiation (dashed curves). Each dataset plotted was the average of 4 specimens.

Figure 7 shows the indentation and recovery data for each group of materials for both immediate and $24 \mathrm{~h}$ initial loading. The maximum of the indentation (Y-scale) range was set at $4 \%$. This corresponds to $160 \mu \mathrm{m}$ penetration into the surface. Immediately following light irradiation, the maximum indentations ranged between $2.5 \%$ to $3.8 \%$, and reduced to $1.41 \%$ to $1.83 \%$ following the $24 \mathrm{~h}$ delay. The residual indentation results seem to follow the trends for maximum indentation, ranging between $1.8 \%$ to $2.8 \%$ at immediate evaluation, which then reduced to 0.57 $\%-0.98 \%$ when measurements were made after $24 \mathrm{~h}$. In general, all materials behaved qualitatively similarly but the measurement time differences (immediate vs. $24 \mathrm{~h}$ ) gave statistically significant differences $(\mathrm{p}<0.05)$.

Indentation curves for the two flowable (low viscosity) formulations were greater in magnitude than for the two non-flowable (regular viscosity) composites. When loaded "immediately" the maximum indentations were notably greater than when loading was deferred for $24 \mathrm{~h}$.

Furthermore, at the time of load-removal, a period of $2 \mathrm{~h}$ had elapsed since initiation of loading. Thus, especially with "immediately" loaded specimens, further matrix polymerization occurred during this interval so that the recovery portions of the curves were reduced in magnitude compared to the response upon initial loading. The maximum and residual indentation data are summarized for each composite group in Figures 8 and 9 and in Table 5. 


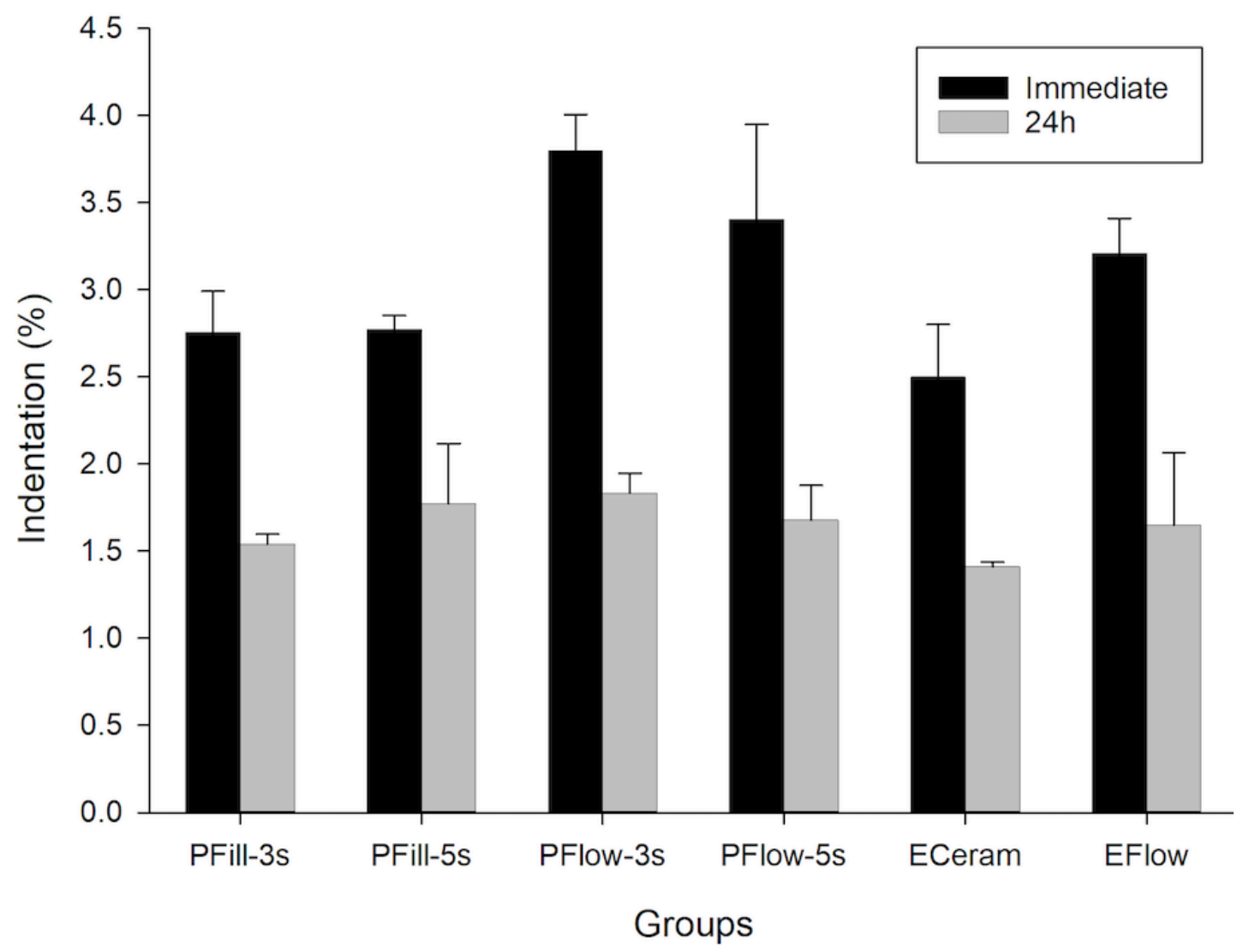

Figure 8: Maximum indentations (\%) within $2 \mathrm{~min}$ (immediately) and $24 \mathrm{~h}$ after irradiation for each group.

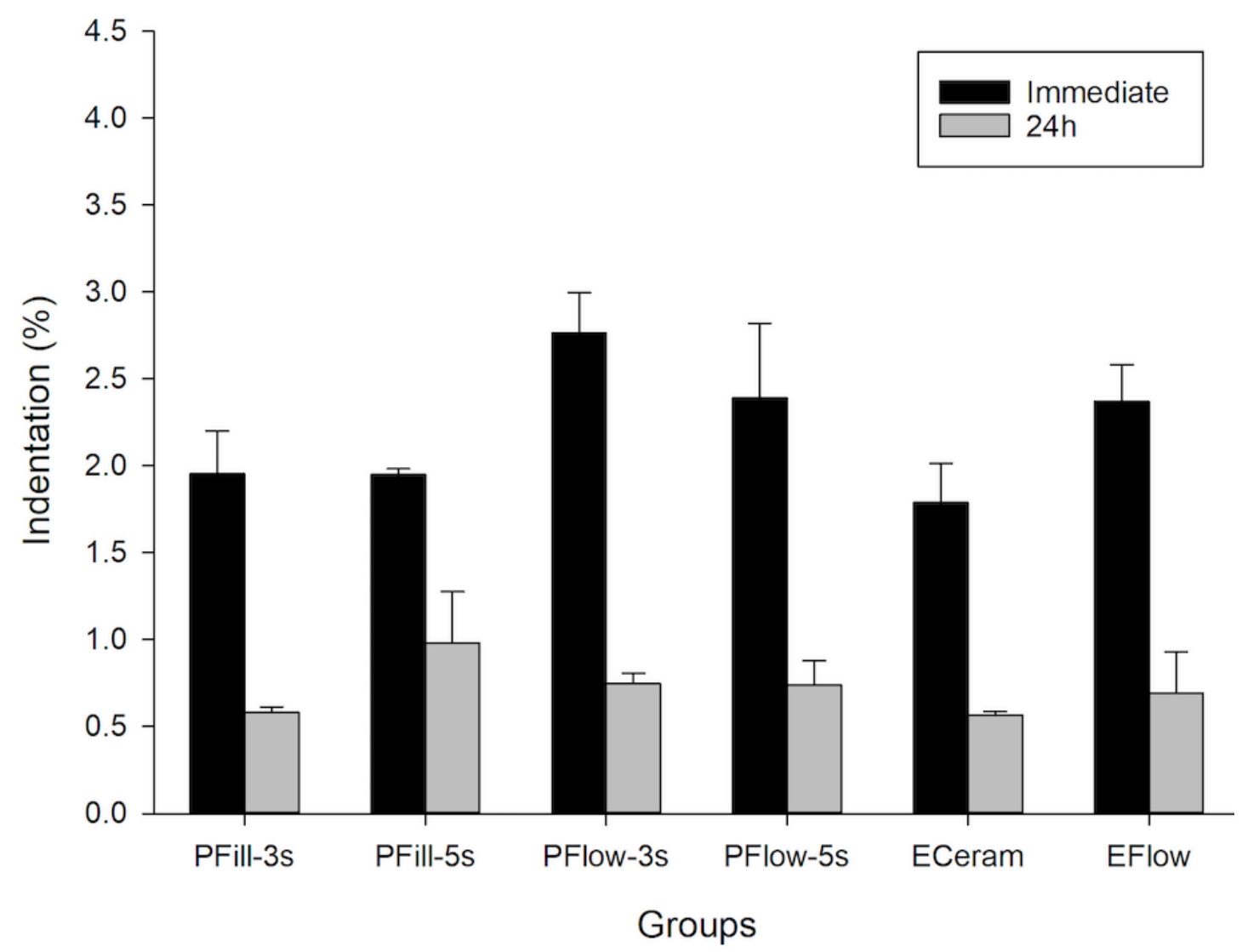

Figure 9: Residual indentations (\%) within 2 min (immediately) and $24 \mathrm{~h}$ after irradiation for each group. 
Table 5. Mean Indentation Creep parameters for 4 materials subjected to 3 irradiation (light cure) protocols (cf. Table 2) following both immediate and delayed indentation.

The parameters are (i) the maximum indentation $(I)$ after $2 \mathrm{~h}$ loading and (ii) the residual indentation after $2 \mathrm{~h}$ unloading. (Standard deviations: in parentheses).

\begin{tabular}{|l|c|c|l|l|l|}
\hline \multicolumn{2}{|c|}{} & \multicolumn{2}{|c|}{$\begin{array}{r}\text { Immediate Indentation } \\
(<2 \mathrm{~min})\end{array}$} & \multicolumn{2}{c|}{$\begin{array}{c}\text { Delayed Indentation } \\
(24 \mathrm{~h})\end{array}$} \\
\hline Material & Irradiation & \multicolumn{1}{|c|}{ max (\%) } & I residual $(\%)$ & I max (\%) & I residual (\%) \\
\hline \multirow{2}{*}{ PFill } & $3 \mathrm{~s}$ & $\mathbf{2 . 7 6}(0.24)^{\mathrm{bcA}}$ & $\mathbf{1 . 9 5}(0.25)^{\mathrm{abA}}$ & $\mathbf{1 . 5 4}(0.06)^{\mathrm{aB}}$ & $\mathbf{0 . 5 8}(0.03)^{\mathrm{bB}}$ \\
\cline { 2 - 6 } & $5 \mathrm{~s}$ & $\mathbf{2 . 7 7}(0.08)^{\mathrm{bcA}}$ & $\mathbf{1 . 9 5}(0.03)^{\mathrm{abA}}$ & $\mathbf{1 . 7 7}(0.34)^{\mathrm{aB}}$ & $\mathbf{0 . 9 8}(0.29)^{\mathrm{bB}}$ \\
\hline \multirow{2}{*}{ PFlow } & $3 \mathrm{~s}$ & $\mathbf{3 . 8 0}(0.21)^{\mathrm{dA}}$ & $\mathbf{2 . 7 7}(0.23)^{\mathrm{cA}}$ & $\mathbf{1 . 8 3}(0.11)^{\mathrm{aB}}$ & $\mathbf{0 . 7 5}(0.06)^{\mathrm{bB}}$ \\
\cline { 2 - 6 } & $5 \mathrm{~s}$ & $\mathbf{3 . 4 1}(0.54)^{\mathrm{cdA}}$ & $\mathbf{2 . 3 9}(0.43)^{\mathrm{bcA}}$ & $\mathbf{1 . 6 8}(0.20)^{\mathrm{aB}}$ & $\mathbf{0 . 7 4}(0.14)^{\mathrm{bB}}$ \\
\hline ECeram & $20 \mathrm{~s}$ & $\mathbf{2 . 5 0}(0.30)^{\mathrm{abA}}$ & $\mathbf{1 . 7 9}(0.23)^{\mathrm{aA}}$ & $\mathbf{1 . 4 1}(0.03)^{\mathrm{aB}}$ & $\mathbf{0 . 5 7}(0.02)^{\mathrm{bB}}$ \\
\hline EFlow & $20 \mathrm{~s}$ & $\mathbf{3 . 2 1}(0.20)^{\mathrm{cdA}}$ & $\mathbf{2 . 3 7}(0.21)^{\mathrm{bcA}}$ & $\mathbf{1 . 6 5}(0.41)^{\mathrm{aB}}$ & $\mathbf{0 . 6 9}(0.24)^{\mathrm{bB}}$ \\
\hline \multicolumn{5}{|l}{} \\
\hline $\begin{array}{l}\text { Significant differences }(\mathrm{p}<0.05) \text { are shown between materials, via different superscript lower- } \\
\text { case letters and between measurement times via different superscript capital letters. }\end{array}$ \\
\hline
\end{tabular}

The experiments showed, from both maximum and residual indentations, that PFill (with either $3 \mathrm{~s}$ or $5 \mathrm{~s}$ modes) and also ECeram (20 s) had generally reduced indentations - and thus superior outcomes - compared to EFlow and PFlow (with either $3 \mathrm{~s}$ or $5 \mathrm{~s}$ modes). The immediate performance of PFill and PFlow composites cured for $3 \mathrm{~s}$ were statistically equivalent to their comparators, ECeram and EFlow cured for $20 \mathrm{~s}(\mathrm{p}>0.05)$. However, $24 \mathrm{~h}$ post-irradiation showed no statistically significant differences among the materials in either maximum or residual indentations $(\mathrm{p}>0.05)$.

Further analysis utilizing independent sample t-tests confirmed a significant difference considering viscosity, with PFlow and EFlow having significantly greater maximum and residual indentations compared to non-flowable PFill and ECeram $(\mathrm{p}<0.05)$.

Furthermore, there was no significant difference in the outcome using the $3 \mathrm{~s}$ or the $5 \mathrm{~s}$ modes ( $>0.05$ ) of the PowerCure LCU-LED. 


\section{Discussion}

The main goal of the present work was to establish a new methodology for addressing critical questions about ultra-rapid photo-polymerized composite materials. Following clinical placement, key requirements for dental restorations include sufficient mechanical integrity during functional occlusal loading. Such clinical "challenges" are normally intermittent in character. However, from a materials science and bioengineering perspective - especially with viscoelastic substances, measuring a "time domain" response to step-function loading /unloading can give representative insights that otherwise would require multiple fatigue loading (on/off) cycles [7, 10, 21].

To achieve this, we have adapted established bulk compressive creep/recovery methodologies [711] by developing an indentation-creep approach based upon a flat-ended cylindrical punch. Because the $1.5 \mathrm{~mm}$ diameter punch is impressed into the center of a $4 \mathrm{~mm}$ diameter composite surface, the specimen can be retained within its rigid stainless-steel split mold. This facilitates rapid transfer of the mold plus irradiated specimen to the loading platform in less than 2 min after $3 \mathrm{~s}$ irradiation. In most cases, the transfer could be completed within $30 \mathrm{~s}$.

In the present work, comparator materials to the PowerCure composites (PFill; PFlow) were chosen from the same manufacturer (ECeram; EFlow). We have also conducted studies with the same methodology on a range of dental composites from other manufacturers. These findings, which exhibit comparable trends to the present study, will be published separately [36]. Moreover, we have made parallel investigations into the kinetics of $\mathrm{C}=\mathrm{C}$ bond conversion by FTIR-ATR methods that will also be reported separately [37].

The irradiation regimes (Table 2) selected were based upon manufacturer's instructions. The delivered energy dose through the $5 \mathrm{~s}$ and $3 \mathrm{~s}$ modes were 10 and $9 \mathrm{~J} / \mathrm{cm}^{2}$, respectively. To some extent, there was an implicit assumption of the general reciprocity hypothesis that: "the same photo-cure outcomes will result from applying essentially constant energy densities despite reciprocal variations in the irradiance and time-period" [33]. However, with our use of the Elipar S10 LCU, the total energy delivery of $24 \mathrm{~J} / \mathrm{cm}^{2}$ was approximately twice that from the Bluephase PowerCure LCU. This was deliberately chosen to provide a severe comparative test of the PowerFill materials. A minimum energy dose of $14 \mathrm{~J} / \mathrm{cm}^{2}$ was suggested [38] to cure bulk-fill resin-composites. However, the outcome depends not only on the energy dose, but on the responsiveness of the photoinitiator system, such as its quantum yield, and other factors.

Surface indentation creep has been applied previously to materials such as solid polymers where unlike the present experiments - the surface-properties were not subject to underlying chemical change. A number of experimental and theoretical analyses have been published [38-42]. Surface measurements have possible limitations, as far as evaluating bulk-fill dental composites are concerned, although it could be possible to invert the steel molds and measure indentations on the specimen surface remote from the irradiated surface. Nevertheless, the magnitudes of the surface indentations or "impressions" [39] are strongly dependent upon the state-of-cure of the underlying bulk material. This contrasts somewhat with the nature of surface microhardness indentations into resin-composites and particularly with nanoindentation [44]. Moreover, Degree of Conversion (DC) measurements of the Powerfill materials at $1 \mathrm{~mm}$ and $4 \mathrm{~mm}$ depths did not exhibit significant differences between the two depths [37]. 
We may critically review some of the measurement conditions of this experiment. Firstly, as with most conventionally photo-cured dental composites, there is a vast change in elastic modulus during the first $10 \mathrm{~s}$ of irradiation [45]. Therefore, with these ultra-fast materials, it was appropriate to probe the surface integrity as rapidly as possible; hence the "immediate" measurement condition. Secondly, a regime of load application for $2 \mathrm{~h}$, followed by a further $2 \mathrm{~h}$ of unloaded recovery, was established by Ruyter [7] for bulk compressive creep and adopted by Watts et al. in many subsequent studies [8-11]. Thirdly, the applied stress was set at $14 \mathrm{MPa}$, which is a comparatively severe condition but here applied equally to each experimental group. Fourthly, the measurement temperature was the constant ambient laboratory temperature of $23^{\circ} \mathrm{C}$. This contrasts with the $37^{\circ} \mathrm{C}$ conditions usually implemented in our bulk compressive creep experiments. To have attempted immediate indentation-creep measurements for ultra-fast photocured composites held at $37^{\circ} \mathrm{C}$ would have introduced complexities, logistical difficulties and potential errors. Moreover, even for bulk creep of composites, the effect of temperature over this small interval was not a major factor [46]. Of course, the polymerization process itself releases exothermic heat, to which the heating effect of the LED-LCU is added. However, the surrounding stainless-steel mold constitutes a heat sink. Furthermore, the moderate thermal diffusivities of dental composites and ultra-short exposures of 3 or $5 \mathrm{~s}$ with the PowerCure LCU must reduce the overall impact of any thermal impulse.

Upon initial loading, 2 - $3 \%$ indentation was measured, depending upon the formulation. Upon unloading at $2 \mathrm{~h}$, elastic recovery was only ca. $1 \%$. The reduction in elastic recovery could be at least partly caused by an increase in elastic modulus in accordance with Equation 1. The maximum indentation-creep under $14 \mathrm{MPa}$, observed with immediate measurements after $3 \mathrm{~s}$ (at 3 $\mathrm{W} / \mathrm{cm}^{2}$ ) irradiation, was less than $150 \mu \mathrm{m}$, even for the flowable formulations. By contrast, delayed indentation by $24 \mathrm{~h}$ gave a maximum "impression" of only $c a .60 \mu \mathrm{m}$. These "worstcase" figures under a severe indentation stress might be compared with loss-of-surface-height during finishing and polishing and/or loss of anatomical form due to occlusal wear. However, the salient feature is that the PowerCure materials exhibited good viscoelastic stability, performing comparably to ECeram and EFlow, but with only half the total energy delivery. This supports their suitability for clinical application.

The post-irradiation time of $24 \mathrm{~h}$ was enough to elicit a significant improvement (reduced indentation) in the surface properties, which agrees with previous studies evaluating surface properties and post-curing [47-50]. Higher indentation-creep was seen in the flowable bulk-fill materials compared to PFill and ECeram. However, this variation was greatly reduced after $24 \mathrm{~h}$, so the null hypotheses were accepted. The higher filler content incorporated in PFill and ECeram (79, $80 \% \mathrm{wt}$, respectively) possibly explains the $c a .25 \%$ lower indentations compared to the flowable PFlow and PFill under immediate loading.

In this report, the PowerCure composites (PFill and PFlow) were compared to the well-established composites (ECeram and EFlow) as the monomer composition and filler content are broadly similar (Table 1). These materials incorporated a photoinitiator based on benzoyl germanium, commercially known as Ivocerin, besides CQ. Main modifications to the PFill and PFlow materials [5] including (i) the elimination of TPO photoinitiator used in ECeram and EFlow, relying on CQ as the main photoinitiator and Ivocerin as a booster (ii) addition-fragmentation chain transfer (AFCT) reagent incorporation with the aim of inducing a more homogenous 
network [5, 34, 35] (iii) tricyclodocane dimethanol dimethacrylate (DCP) and propoxylated Bisphenol A dimethacrylate as added monomers.

The two mathematical functions, illustrated in Figure 6 for PFill, are able to provide close fits, respectively, to either indentation-creep data for immediate loading (equation 8; Table 3) or for delayed loading (Equation 12; Table 4). KWW or stretched-exponential behavior is characteristic of complex materials in which hierarchical structures exist $[26,27]$. The fractional exponents $(\beta)$ were low and in the range 0.16 to 0.23 .

Fancey [29] considered a complex and generalized Voigt model with Voigt elements connected in series (cf. Figure 2) with the aim of applicability to broad timescales. However, he noted that in these models, viscoelastic deformations are considered to vary smoothly i.e continuously with time. He considered an alternative approach in which viscoelastic changes are suggested to occur through discrete incremental jumps, via activated mechanical latches. On a molecular level, the phenomenon could be envisaged as segments of molecules jumping between positions of relative stability. This is certainly the concept accepted within the advanced science of polymers and networks. A link that has been proposed [51] between the KWW function and the potential energy barrier relationship as developed by Eyring et al. [52], since the latter relates the motion of matter to molecular jumps. The $\mathbf{K W W}$ function is shown to be an approximation to the potential energy barrier model [51]. Thus, during any photo-cure process, network links are formed - and some may also be broken under applied stress, particularly in a RAFT or AFCT system. Nevertheless, sufficient matrix-network connectivity is clearly established in the final polymerized materials.

\section{Conclusions}

The extent of immediate surface rigidity and developing viscoelastic characteristics of ultra-rapid photo-polymerized dental composites can be measured by a macroscopic indentation-creep method, following $3 \mathrm{~s}$ or $5 \mathrm{~s}$ exposures to irradiances, respectively, of 3 or $2 \mathrm{~W} / \mathrm{cm}^{2}$. The new methodology is described in detail. For rapid-irradiated PowerCure composites under $14 \mathrm{MPa}$ immediate stress, the indentation-creep exhibited stretched-exponential kinetics with magnitudes after $2 \mathrm{~h}$ less than $150 \mu \mathrm{m}$. When the stress application was delayed for $24 \mathrm{~h}$, the indentation magnitudes were $c a$. $60 \mu \mathrm{m}$. Completely comparable indentation/time profiles were exhibited by well-established composite formulations following $1.2 \mathrm{~W} / \mathrm{cm}^{2}$ irradiation for $20 \mathrm{~s}$. As judged by the experimental indentation-creep methodology, the PowerCure system and materials demonstrated an acceptable level of polymerization/property performance, despite the ultra-short irradiation times, evidencing their suitability for clinical application.

\section{Author Contributions}

DCW conceived and commissioned the instrumental design, methods and theoretical analysis, guided the experiments and wrote the final manuscript to which both authors gave approval. HA performed the experiments, collated the data, performed statistics, contributed to the interpretation of results and to manuscript writing.

\section{Acknowledgements}

The authors thank Ivoclar-Vivadent AG for provision of composite materials. The authors declare no conflict of interest. 


\section{Highlights}

- The rapidly developing surface integrity of ultra-fast (3 s) photo-cure composites can be probed "immediately" by a new macroscopic indentation-creep methodology.

- PowerCure system and materials demonstrated an acceptable level of polymerization/property performance, despite the ultra-short irradiation times, as judged by this experimental criterion.

\section{References}

1. Maas MS, Alania Y, Natale LC, Rodrigues MC, Watts DC, Braga RR. Trends in restorative composites research: what is in the future? Braz Oral Res 2017; 31, supl. 1.

2. Watts DC, Amer O, Combe EC. Characteristics of visible-light-activated composite systems. Br Dent J, 1984; 156: 209-215.

3. Rueggeberg FA. State-of-the-art: dental photocuring - a review. Dent Mater 2011; 27: 39-52.

4. Jandt KD, Mills RW. A brief history of LED photopolymerization. Dent Mater 2013; 29: 605617.

5. Ilie N, Watts DC. Outcomes of ultra-fast ( $3 \mathrm{~s})$ photo-cure in a RAFT-modified resincomposite. Dent Mater 2020; 36: 570-579.

6. Stansbury JW. Dimethacrylate network formation and polymer property evolution as determined by the selection of monomers and curing conditions. Dent Mater 2012; 28:13-22.

7. Odén A, Ruyter IE, Øysaed H. Creep and recovery of composites for use in posterior teeth during static and dynamic compression. Dent Mater 1988; 4:147-150

8. El Hejazi AA, Watts DC. Creep and viscoelastic recovery of cured and secondary-cured composites and resin-modified glass-ionomers. Dent Mater 1999; 15: 138-143.

9. El-Safty S, Silikas N, Watts DC. Creep deformation of restorative resin-composites intended for bulk-fill placement. Dent Mater 2012; 28: 928-935.

10. Kaleem M, Masouras K, Satterthwaite JD, Silikas N, Watts DC. Viscoelastic stability of resincomposites under static and dynamic loading. Dent Mater 2012; 28: e15-e18.

11. Al-Ahdal K, Silikas N, Watts DC. Development of viscoelastic stability of resin-composites incorporating novel matrices. Dent Mater 2015; 31: 1561-1566.

12. Yang S, Zhang YW, Zeng K. Analysis of nanoindentation creep for polymeric materials. $J$. Appl. Phys. 2004; 95(7): 3655-3666.

13. El-Safty S, Akhtar R, Silikas N, Watts DC. Nanomechanical properties of dental resincomposites. Dent Mater 2012; 28: 1292-1300.

14. El-Safty S, Silikas N, Akhtar R, Watts DC. Nanoindentation creep versus bulk compressive creep of dental resin-composites. Dent Mater 2012; 28: 1171-1182.

15. Ilie N, Hickel R, Watts DC. Spatial and cure-time distribution of dynamic-mechanical properties of a dimethacrylate nano-composite. Dent Mater 2009; 25: 411-418.

16. Boussinesq J, Applications des Potentiels à l'Étude de l'Équilibre et du Mouvement des Solides Élastiques, Gauthier-Villars, Paris, 1885; also see Todhunter I and Pearson K, History of the Theory of Elasticity, 2, 237 et seq. Cambridge University Press, 1893.

17. Galin LA, Kontakn'e Zadachi Teorii Uprugosti. Gos. Izdat. Tech.-Teor. Liter., Moscow. (1953).

18. Harding JW, Sneddon IN. The elastic stresses produced by the indentation of the plane surface of a semi-infinite elastic solid by a rigid punch. Proc. Camb. Phil. Soc. 1945; 41: 16-26.

19. Sneddon IN. Boussinesq's problem for a flat-ended cylinder. Proc. Camb. Phil. Soc. 1946; 44: 29-39.

20. Sneddon IN. The relation between load and penetration in the axisymmetric Boussinesq problem for a punch of arbitrary profile. Int. J. Eng. Sci. 1965; 3: 47-57.

21. Lakes R. Viscoelastic Materials. Cambridge University Press. 2009.

22. Findley WN, Lai JS, Onaran K. Creep and relaxation of nonlinear viscoelastic materials. Dover edition, 1989.

23. Kohlrausch R. Theorie des electrischen Rückstandes in der leidener flasche. Annalen der Physic. 1854; 167(2):179-214. 
24. Williams G, Watts DC. Non-symmetrical dielectric relaxation behaviour arising from a simple empirical decay function. Trans Faraday Soc 1970; 66: 80-85.

25. Williams G, Watts DC, Dev SB, North AM. Further considerations of non-symmetrical dielectric relaxation behaviour arising from a simple empirical decay function. Trans Faraday Soc 1971; 67: 1323-1335.

26. Palmer RG, Stein DL, Abrahams E, Anderson PW. Models of hierarchically constrained dynamics for glassy relaxations. Phys Rev Lett 1984; 53: 958-961.

27. Berry GC, Plazek DJ, On the use of stretched-exponential functions for both linear viscoelastic creep and stress relaxation. Rheol. Acta 1997; 36: 320-329.

28. Cardona M, Chamberlin RV, Marx W. The history of the stretched exponential function, Annalen der Physik 2007; 16(12): 842-845.

29. Fancey KS. A mechanical model for creep, recovery and stress relaxation in polymeric materials. J. Mater. Sci. 2005; 40: 4827-4831.

30. Wydra J, Cramer N, Stansbury J, Bowman C. The reciprocity law concerning light-dose relationships applied to BisGMA/TEGDMA photopolymers: theoretical analysis and experimental characterization. Dent Mater 2014; 30: 605-612.

31. Hadis M, Leprince JG, Shortall AC, Devaux J, Leloup G, Palin WM. High irradiance curing and anomalies of exposure reciprocity law in resin-based materials. $J$ Dent 2011; 39: 549-557.

32. Randolph LD, Palin WM, Watts DC, Genet M, Devaux J, Leloup G, Leprince JG. The effect of ultra-fast photopolymerisation of experimental composites on shrinkage stress, network formation and pulpal temperature rise. Dent Mater, 2014; 30: 1280-1289.

33. Sadeghyar A, Watts DC, Schedle A. Limited reciprocity in curing efficiency of bulk-fill resin-composites. Dent Mater 2020; 36(8): 997-1008.

34. Barner-Kowollik C (Ed.) Handbook of RAFT Polymerization. Wiley-VCH Verlag GmbH \& Co. KaGaA. Weinheim, 2008.

35. Gorsche C, Griesser M, Gescheidt G, Moszner N, Liska R. $\beta$-Allyl sulfones as addition-fragmentation chain transfer reagents: A tool for adjusting thermal and mechanical properties of dimethacrylate networks. Macromolecules 2014; 47: 7327-7336.

36. Algamaiah H, Silikas N, Watts DC. Surface viscoelastic integrity of photo-polymerized resincomposites. In preparation.

37. Algamaiah H, Silikas N, Watts DC. Conversion kinetics of rapid photo-polymerized resin composites. Dent Mater 2020; 36:

38. Daugherty MM, Lien W, Mansell MR, Risk DL, Savett DA, Vandewalle KS. Effect of highintensity curing lights on the polymerization of bulk-fill Composites. Dent Mater, 2018; 34: 1531-1541.

39. Chu SNG, Li JCM. Impression creep; a new creep test. J. Mater. Sci. 1977; 12: 2200-2208.

40. Hill R. Similarity analysis of creep indentation tests. Proc. R. Soc. Lond. A, 1992; 436: 617630.

41. Berthaud P, G'Sell C, Hiver J-M. Elastic-plastic indentation creep of glassy poly(methy methacrylate) and polystyrene: characterization using uniaxial and indentation tests. J. Phys. D: Appl. Phys. 1999; 32: 2923-2932.

42. Beake B. Modelling indentation creep of polymers: a phenomenological approach. J. Phys. D: Appl. Phys. 2006; 39: 4478-4485.

43. Quadrini F, Squeo EA, Guglielmotti A. Indentation creep of polymers. I. Experimental. Polym. Eng. Sci. 2010; 50: 2431-2439.

44. Yang S, Zhang YW, Zeng K. Analysis of nanoindentation creep for polymeric materials. $J$. Appl. Phys. 2004; 95(7): 3655-3666.

45. Kim MH, Min SH, Ferracane J, Lee IB. Initial dynamic viscoelasticity change of composites during light curing. Dent Mater, 2010; 26: 463-470.

46. El-Safty S, Silikas N, Watts DC. Temperature dependence of creep behaviour of dental resincomposites. J Dent 2012; 41: 287-296.

47. Watts DC, Amer OM, Combe EC. Surface hardness development in light-cured composites. Dent Mater, 1987; 3: 265-269. 
48. Par M, Gamulin O, Marovic D, Klaric E, Tarle Z. Effect of temperature on post-cure polymerization of bulk-fill composites. J Dent, 2014; 42: 1255-1260.

49. Al-Ahdal K, Ilie N, Silikas N, Watts DC. Polymerization kinetics and impact of post polymerization on the degree of conversion of bulk-fill resin-composite at clinically relevant depth. Dent Mater, 2015; 31: 1207-1213.

50. Kaiser C, Price RB. Effect of time on the post-irradiation curing of six resin-based composites. Dent Mater 2020; 36:

51. Dobreva A, Gutzow I, Schmelzer J. Stress and time dependence of relaxation and the Kohlrausch stretched exponent formula. J. Non-Cryst. Solids 1997; 209: 257 -263.

52. Glasstone S, K. Laidler KH, Eyring H. The Theory of Rate Processes: McGraw-Hill, New York, 1941 p. 480. 